\title{
Promoting youth mental health
}

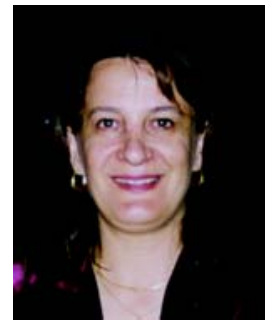

Tania Janusic Deputy Edito

tjanusic@mja.com.au doi: 10.5694/mjal2.c0521 iven that prevention is better than cure, it is not surprising that we search for an effective way of intervening to assist young people at high risk of psychosis. Unfortunately, unlike some chronic diseases, it is still unclear when, how and in whom to intervene. Especially in the very young, we are in the early stages of understanding how psychiatric syndromes evolve and whether early symptoms are indicative of incipient illness or are transitory phenomena. In the Opposing Views articles in this issue of the Journal, Yung (page 556) advocates intervention to identify "ultra-high risk" individuals with "subthreshold psychotic symptoms" who are not coping and are seeking help. Castle (page 557), however, wonders about who exactly qualifies for treatment and why, and believes the risk of mislabelling someone as psychotic — with all its personal and vocational sequelae - outweighs the as yet unproven benefits of early intervention.

Moreover, the diagnostic category of "risk syndrome for first psychosis" - proposed for inclusion in the next edition of the Diagnostic and statistical manual of mental disorders (DSM-5), due out in 2013 - is in question, as discussed by Fitzgerald (page 549). He warns about broadening the diagnostic categories and the potential harm from treating people who, regardless of intervention, would not have transitioned to psychosis. In discussing the DSM-5, he also emphasises the difficulty of knowing when psychological reactions to life events such as bereavement are a disorder, and when they are a normal and possibly adaptive response. Do we assign a diagnostic label in order to identify and treat the severely distressed, but in turn run the risk of overmedicalising and overtreating others?

Even for established conditions, making a careful and correct diagnosis is central to delivering appropriate care.
Given the implications of the treatment they deliver, it is easy to understand why the multidisciplinary staff at the gender identity disorder (GID) clinic in Melbourne spend a lot of time confirming a diagnosis of GID. Although it resolves in most children and adolescents, about $44 \%$ of those referred to the clinic for gender dysphoria were pubertal with persistent GID and were considered eligible for hormone treatment. Once recommended, hormone treatment needs to be agreed to by the patient and their family. Permission is then sought from the Family Court. While there is little evidence from longitudinal studies on mental health outcomes among patients who undergo treatment, the study by Hewitt and colleagues (page 578) presents early descriptive data on a previously neglected group.

Another hotly debated issue is the impact of exposure to internet pornography on adolescent mental health. Guy and colleagues (page 546) discuss the need for "a new generation of cohort studies" in this area. One in three 9-16-year-olds have reported seeing sexual material online, and cross-sectional data show an association between exposure to sexual material and earlier onset of sexual activity. Given that young people seem to find a way to overcome internet filters, it is certainly time for more open discussion with adolescents about sexual material on the internet.

In an area of medicine that is often data-poor, emotive and politicised, many diagnostic and management decisions are made on a case-by-case basis in imperfect circumstances. Clearly, there is an urgent need for robust evidence to inform when and how to intervene and provide care for the mental health of young people. Whether we give them a label or not, we need to take more care.

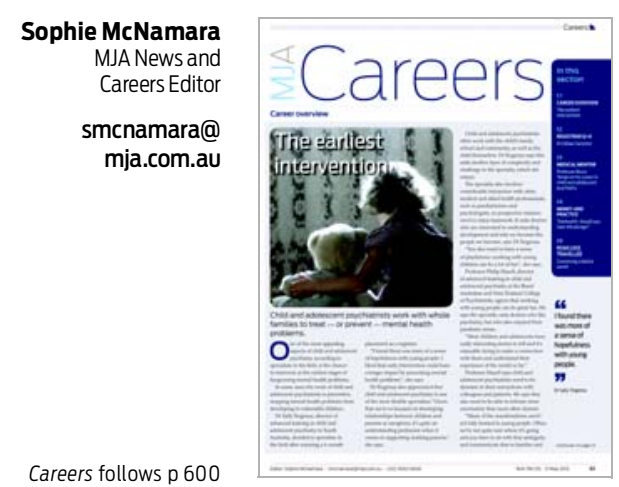

Careers follows p 600

\section{The earliest intervention}

Child and adolescent psychiatrists often work with children before mental health conditions have fully formed. Specialists working in the field say this opportunity to intervene early and improve quality of life for decades to come is what makes their specialty so rewarding. In this issue of MJA Careers, we talk to several child and adolescent psychiatrists, ranging from a registrar (page
C2), to a prominent, experienced psychiatrist who has had a big impact on the field (page C5). Also in this issue, the Money + Practice section looks at the practical side of telehealth, explaining what is involved in making your first telehealth consultation (page C6). We talk to specialists who are already using telehealth to expand their patient base beyond their local area. 